\title{
Aceruloplasminemia: A Case Report and Review of a Rare and Misunderstood Disorder of Iron Accumulation
}

\author{
Chimaobi M. Anugwom ${ }^{1}$, Carlos G. Moscoso ${ }^{2}$, Nicholas Lim ${ }^{1}$, Mohamed Hassan ${ }^{1}$ \\ 1. Gastroenterology, Hepatology and Nutrition, University of Minnesota, Minneapolis, USA 2. Gastroenterology, \\ University of Minnesota, Minneapolis, USA
}

Corresponding author: Chimaobi M. Anugwom, anugw001@umn.edu

\begin{abstract}
Aceruloplasminemia is a rare disorder of iron accumulation inherited in an autosomal recessive fashion. It commonly presents as chronic microcytic anemia, and then progresses to signs and symptoms that are due to the accumulation of iron in multiple organs such as the brain, liver, pancreas, and thyroid. We present an asymptomatic patient with a history of microcytic anemia, who was evaluated for abnormal liver enzymes, and ultimately diagnosed with aceruloplasminemia.
\end{abstract}

Categories: Genetics, Gastroenterology

Keywords: ceruloplasmin transferrin, liver damage, genetic metabolic disorders

\section{Introduction}

Aceruloplasminemia is a rare autosomal recessive disorder of iron metabolism resulting in multi-systemic iron overload. It typically presents with mild chronic microcytic anemia, variable hepatic dysfunction, neuropsychiatric disturbances, and other sequelae of iron overload such as pancreas and thyroid disease. We present a patient with abnormal liver function tests and chronic microcytic anemia who was ultimately diagnosed with aceruloplasminemia.

\section{Case Presentation}

A 51-year-old female with a medical history of chronic microcytic anemia (baseline hemoglobin 10-11 g/dL) presented to the emergency department with new-onset post-prandial right upper quadrant pain. Laboratory evaluation showed an aspartate aminotransferase (AST) of $361 \mathrm{U} / \mathrm{L}$, alanine aminotransferase (ALT) of $91 \mathrm{U} / \mathrm{L}$, alkaline phosphatase (ALP) of $162 \mathrm{U} / \mathrm{L}$, and total bilirubin of 0.6 $\mathrm{mg} / \mathrm{dL}$. Abdominal ultrasound showed cholelithiasis without cholecystitis or biliary duct dilation. Magnetic resonance cholangiopancreatography (MRCP) was obtained and was also negative for intra- or extrahepatic biliary dilation. The patient was admitted to the hospital and her liver enzymes continued to rise with AST 1422 U/L, ALT 1393 U/L, ALP 197 U/L, and total bilirubin of $0.3 \mathrm{mg} / \mathrm{dL}$. Laparoscopic cholecystectomy was performed without complication and the patient was discharged home.

Review began 10/20/2020 Review ended 11/13/2020 Published 11/23/2020

\section{๑) Copyright 2020}

Anugwom et al. This is an open access article distributed under the terms of the Creative Commons Attribution License CC-BY 4.0., which permits unrestricted use, distribution, and reproduction in any medium, provided the original author and source are credited.
The patient was seen in the hepatology clinic with resolution of abdominal pain but persistently elevated liver enzymes. She was not jaundiced on physical examination, had no asterixis, and had no skin evidence of chronic liver disease such as spider angioma or palmar erythema. Additional laboratory evaluation revealed negative serologies for hepatitis A, B, and C, negative serologies for autoimmune hepatitis, and primary biliary cholangitis. Iron studies showed a low serum iron of $20 \mathrm{mcg} / \mathrm{dL}$, a low iron saturation of $6 \%$, and elevated serum ferritin of $7133 \mathrm{ng} / \mathrm{ml}$. Subsequent testing for hereditary hemochromatosis was negative for mutations C282Y, H63D, and S65C. To ensure a full evaluation was completed for abnormal liver enzymes, serum copper and ceruloplasmin were also checked and were found to be undetectable. In addition, her 24hour urine copper was noted to be $<1 \mathrm{mcg} / \mathrm{dL}$. Magnetic resonance imaging (MRI) of the liver revealed significant iron deposition in the liver. Percutaneous liver biopsy was performed, and histopathology showed marked hemosiderosis with accumulation of iron in hepatic parenchymal cells but no hepatic fibrosis.

The patient was then sent to ophthalmology for slit lamp examination which revealed the marginal appearance of Kayser-Fleischer rings. MRI of the brain was obtained to evaluate for iron deposition in the brain and was significant for mild excess mineral deposition in the basal ganglia, mid-brain, and dentate nuclei (Figures 1-2). Based on these findings, a diagnosis of aceruloplasminemia was entertained and the patient was referred to a genetic counsellor for discussion and testing. Using next-generation sequencing, two variants were detected in the CP gene - c.2149C>T (p.Gln717X), located on exon 12, and c.2972T >C (p.Ile991Thr), located on exon 17, both adjudged to be pathogenic. Next-generation sequencing was done with molecular confirmation of a diagnosis of autosomal recessive aceruloplasminemia. 


\section{Cureus}

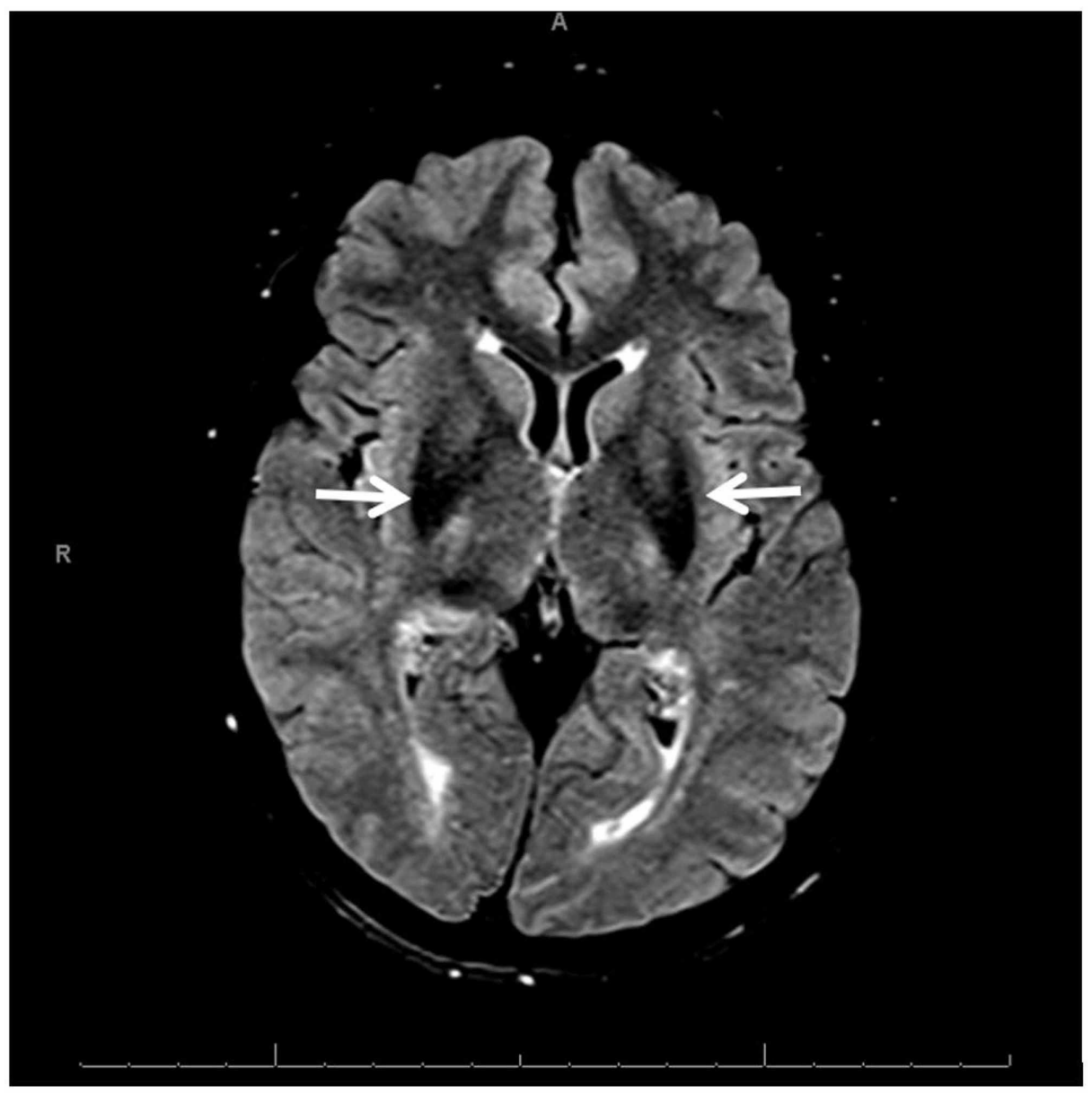

FIGURE 1: Brain magnetic resonance T2-flair images with prominent hypo-intense signal in the region of the basal ganglia (white arrows); A: anterior, R: right side 


\section{Cureus}

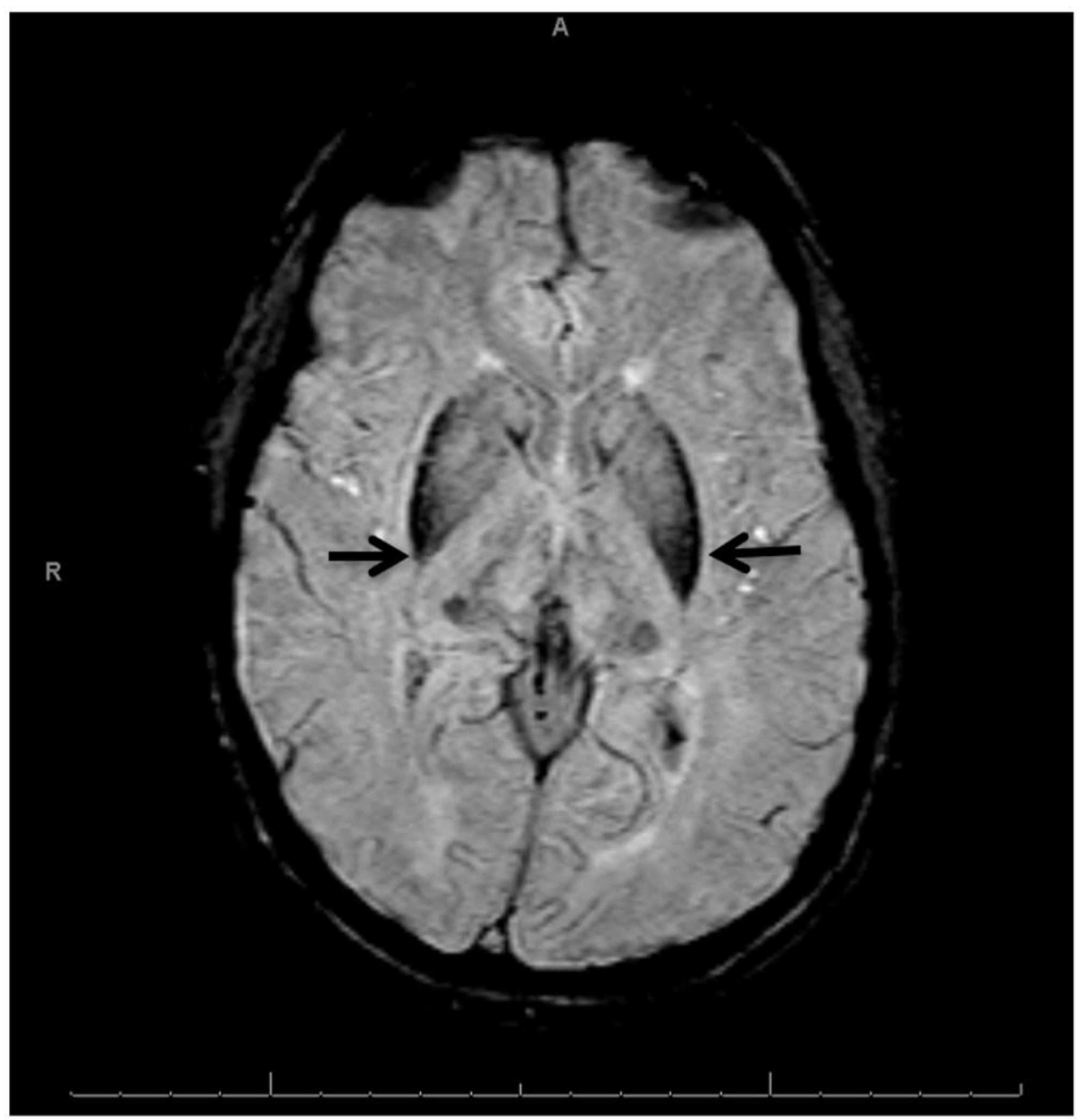

\section{FIGURE 2: Brain magnetic resonance susceptibility weighted images (SWI) showing mildly increased iron deposition in the basal ganglia (black arrows); A: anterior, R: right side}

The patient was referred for neurology and hematology consultations, as well as neuropsychological testing, all of which revealed no additional significant findings. Laboratory testing for thyroid dysfunction and diabetes mellitus have both been negative. Despite chronic mild microcytic anemia, the patient remains asymptomatic. Iron chelation therapy was discussed with her but she is being followed closely in the clinic without any therapy plans at this time. She was adequately counseled on the possibility of neurological symptoms in the future. There was no known diagnosis of aceruloplasminemia in her family and her family members have been referred for genetic counselling for discussion regarding further genetic testing.

\section{Discussion}

Ceruloplasmin is a ferroxidase that can bind up to six atoms of copper. It plays a vital role in iron homeostasis, especially in the brain where it performs a neuroprotective function by safely oxidizing ferrous iron without the generation of free radicals, promoting iron efflux and reducing oxidative

damage [1]. Although ceruloplasmin binds up to 95\% of the body's total copper in the plasma and is needed for copper to maintain its three-dimensional structure and enzymatic activity, it is not involved in copper metabolism [2].

Aceruloplasminemia is the hereditary absence of ceruloplasmin. It is a rare autosomal recessive disorder that occurs due to biallelic mutations in the CP gene located in chromosome locus 3q24-25 which result in systemic iron overload involving the eyes, pancreas, liver, and brain [3,4].

Ceruloplasmin, and other multicopper ferroxidases (MCFs) such as hephaestin, are vital to iron efflux from enterocytes, macrophages, hepatocytes, and neuronal cells [5]. Hephaestin is strongly expressed in the small intestine, whereas ceruloplasmin is located in other cells including those of the kidney, liver, and brain [6]. This function in the brain, where it plays a major role in regulating the uptake of iron by neuronal cells, thus reducing iron accumulation and oxidative stress, suggests that it confers a neuroprotective function [7]. In aceruloplasminemia, the inability to oxidize ferrous iron and promote binding to transferrin results in 
decreased iron delivery for erythropoiesis, which may be the putative mechanism for microcytic anemia in these patients [8]. Iron accumulation in the brain, beta cells of the pancreatic islets, and other visceral organs results in significant tissue damage, and this occurs in tandem with the loss of the antioxidant properties of ceruloplasmin, leading to unchecked production of deleterious oxygen species and resultant free-radical injury $[9,10]$. This mechanism has been demonstrated in studies where markers of lipid peroxidation from oxidative stress have been measured in patients with aceruloplasminemia [11].

Based on studies in Japan done by Miyajima et al., the estimated disease prevalence of aceruloplasminemia is estimated to be 1 in 2 million individuals born from non-consanguineous marriages [12], with most patients presenting between the ages 20 and 60. Outside Japan, however, the prevalence is largely unknown.

Miyajima described the classic triad of retinal degeneration, dementia, and diabetes based on observation of Japanese patients with aceruloplasminemia [13]. However, a much broader clinical presentation can be seen with cerebellar dysfunction, involuntary movements such as chorea, apathy, and memory loss being more common presenting symptoms [14]. Late-onset diabetes mellitus has been documented as a presenting syndrome, and this precedes the development of neurological symptoms [10]. Central hypothyroidism can occur from reduced production of thyrotropin-releasing hormone (TRH) related to iron deposition in the hypothalamus, but no other endocrine diseases have been reported to occur in patients with aceruloplasminemia [15].

Diminished or undetectable ceruloplasmin levels are also very suggestive of this disease although Wilson disease and Menkes disease must be considered in the differential diagnosis in the context of this finding [16]. The laboratory findings of microcytic anemia, low transferrin saturation, and high serum ferritin, as were apparent in our patient, are very common findings in aceruloplasminemia [14]. Her elevated liver enzymes were likely a result of biliary disease, making her diagnosis of aceruloplasminemia incidental. A retrospective review demonstrated that anemia is seen in $80 \%$ of patients and is often present in childhood, long before other neurologic symptoms develop [14,17]. Based on this clinical course, our patient remains at increased risk for neurological complications though it is difficult to predict the timing of development of these manifestations.

Liver biopsy in these patients may be misleading as the presence of iron deposition in liver parenchymal cells may suggest HFE-hemochromatosis. However, cirrhosis from aceruloplasminemia is unlikely to occur and overt hepatic dysfunction and liver failure is rare [18]. Brain imaging may be elucidative and evaluation with MRI may demonstrate neurodegeneration in the thalamus, basal ganglia, and cerebellum [19]. Unequivocal diagnosis of aceruloplasminemia is achieved with genetic testing showing biallelic mutations in the CP gene. Since many cases of aceruloplasminemia are asymptomatic, diagnosis is dependent on a high index of suspicion especially with positive family history and mild laboratory abnormalities such as microcytic anemia.

There is limited data on the management of aceruloplasminemia and most available literature is based on case reports and case series. The mainstay of treatment is iron chelation, and though quite helpful in reducing iron in the liver parenchyma, it has a questionable impact on neurological manifestations [14]. Deferiprone is a frequently used chelating agent, which in contrast with deferoxamine and deferasirox, crosses the blood-brain barrier [16]. Iron chelation therapy may also be used in tandem with fresh frozen plasma to restore serum ceruloplasmin. The use of vitamin $\mathrm{E}$ or zinc has been studied with both medications having fleeting benefits [14]. Hayashida et al. published a case report demonstrating improved neurological status in a patient with aceruloplasminemia using minocycline [20]. Recombinant ceruloplasmin has been used in mice, revealing improved neurological function and the salvage of ferroxidase activity, however, this has not been replicated in humans with aceruloplasminemia [17].

\section{Conclusions}

In conclusion, while our patient developed abnormal liver enzymes due to symptomatic biliary disease, the diagnosis of aceruloplasminemia was incidental. Although many cases of aceruloplasminemia are asymptomatic and depend on a high degree of suspicion to obtain a diagnosis, the presence of iron overload, in the setting of microcytic anemia and absent ceruloplasmin, should prompt genetic testing for this rare condition.

\section{Additional Information \\ Disclosures}

Human subjects: Consent was obtained by all participants in this study. Conflicts of interest: In compliance with the ICMJE uniform disclosure form, all authors declare the following: Payment/services info: All authors have declared that no financial support was received from any organization for the submitted work. Financial relationships: All authors have declared that they have no financial relationships at present or within the previous three years with any organizations that might have an interest in the submitted work. Other relationships: All authors have declared that there are no other relationships or activities that could appear to have influenced the submitted work. 


\section{References}

1. Bento I, Peixoto C, Zaitsev VN, Lindley PF: Ceruloplasmin revisited: structural and functional roles of various metal cation-binding sites. Acta Cryst. 2007, 63:240-248. 10.1107/s090744490604947x

2. Gitlin JD, Schroeder JJ, Lee-Ambrose LM, Cousins RJ: Mechanisms of caeruloplasmin biosynthesis in normal and copper-deficient rats. Biochem J. 1992, 282:835-839. 10.1042/bj2820835

3. Miyajima H: Aceruloplasminemia. Neuropathology. 2015, 35:83-90. 10.1111/neup.12149

4. Yoshida K, Furihata K, Takeda S, et al.: A mutation in the ceruloplasmin gene is associated with systemic hemosiderosis in humans. Nat Genet. 1995, 9:267-272. 10.1038/ng0395-267

5. Kosman DJ: FET3P, ceruloplasmin, and the role of copper in iron metabolism . Adv Protein Chem. 2002, 60:221-269. 10.1016/s0065-3233(02)60055-5

6. Jiang B, Liu G, Zheng J, et al.: Hephaestin and ceruloplasmin facilitate iron metabolism in the mouse kidney . Sci Rep. 2016, 6:39470. 10.1038/srep39470

7. Qian ZM, Ke Y: Rethinking the role of ceruloplasmin in brain iron metabolism . Brain Res Rev. 2001, 35:287294. 10.1016/s0165-0173(01)00056-X

8. Lawen A, Lane DJR: Mammalian iron homeostasis in health and disease: uptake, storage, transport, and molecular mechanisms of action. Antioxid Redox Signal. 2013, 18:2473-2507. 10.1089/ars.2011.4271

9. Patel BN, Dunn RJ, Jeong SY, Zhu Q, Julien JP, David S: Ceruloplasmin regulates iron levels in the CNS and prevents free radical injury. J Neurosci. 2002, 22:6578-6586. 10.1523/JNEUROSCI.22-15-06578.2002

10. Miyajima H, Takahashi Y, Shimizu H, Sakai N, Kamata T, Kaneko E: Late onset diabetes mellitus in patients with hereditary aceruloplasminemia. Intern Med. 1996, 35:641-645. 10.2169/internalmedicine.35.641

11. Miyajima H, Adachi J, Kohno S, Takahashi Y, Ueno Y, Naito T: Increased oxysterols associated with iron accumulation in the brains and visceral organs of acaeruloplasminaemia patients. QJM. 2001, 94:417-422. 10.1093/qjmed/94.8.417

12. Miyajima H, Nishimura Y, Mizoguchi K, Sakamoto M, Shimizu T, Honda N: Familial apoceruloplasmin deficiency associated with blepharospasm and retinal degeneration. Neurology. 1987, 37:761. 10.1212/wnl.37.5.761

13. Miyajima H, Takahashi Y, Kono S: Aceruloplasminemia, an inherited disorder of iron metabolism . Biometals. 2003, 16:205-213. 10.1023/a:1020775101654

14. Pelucchi S, Mariani R, Ravasi G, et al.: Phenotypic heterogeneity in seven Italian cases of aceruloplasminemia. Parkinsonism Relat Disord. 2018, 51:36-42. 10.1016/j.parkreldis.2018.02.036

15. Klomp LWJ, Gitlin JD: Expression of the ceruloplasmin gene in the human retina and brain: implications for a pathogenic model in aceruloplasminemia. Hum Mol Genet. 1996, 5:1989-1996. 10.1093/hmg/5.12.1989

16. Kono S: Aceruloplasminemia: an update. Int Rev Neurobiol. 2013, 110:125-151. 10.1016/b978-0-12-4105027.00007-7

17. Zanardi A, Conti A, Cremonesi M, et al.: Ceruloplasmin replacement therapy ameliorates neurological symptoms in a preclinical model of aceruloplasminemia. EMBO Mol Med. 2018, 10:91-106. 10.15252/emmm.201708361

18. Hellman NE, Schaefer M, Gehrke S, Stegen P, Hoffman WJ, Gitlin JD, Stremmel W: Hepatic iron overload in aceruloplasminaemia. Gut. 2000, 47:858-860. 10.1136/gut.47.6.858

19. McNeill A, Birchall D, Hayflick SJ, et al.: T2* and FSE MRI distinguishes four subtypes of neurodegeneration with brain iron accumulation. Neurology. 2008, 70:1614-1619. 10.1212/01.wnl.0000310985.40011.d6

20. Hayashida M, Hashioka S, Miki H, Nagahama M, Wake R, Miyaoka T, Horiguchi J: Aceruloplasminemia with psychomotor excitement and neurological sign was improved by minocycline (case report). Medicine. 2016, 95:e3594. 10.1097/md.0000000000003594 